\title{
Neue Wege im Flussgebietsmanagement
}

\begin{abstract}
Mit der Ende 2000 in Kraft getretenen EG-Wasserrahmenrichtlinie wird der Gewässerschutz auf europäischer Ebene umfassend neu geregelt. Auch bei der Umsetzung der Richtlinie werden neve Wege beschritten. In Deutschland stellt aufgrund der föderalen Struktur die Implementierung eine große Herausforderung dar. Hierzu werden unter anderem das Wasserhaushaltsgesetz sowie die 16 Landeswassergesetze koordiniert novelliert. Eine Vielzahl von Arbeitsgruppen kümmert sich um die fachliche Umsetzung.
\end{abstract}

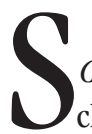
Von Holger Brackemann, Christiane Markard und Jörg Rechenberg chon seit Mitte der 70er Jahre wird auf EGEbene Gewässerschutz betrieben. Allerdings waren die Richtlinien bisher an einzelnen Nutzungen - zum Beispiel Anforderungen zum Schutz von Muschelgewässern, Fischgewässern, Trinkwassergewinnung und Badegewässern - orientiert und nicht aufeinander abgestimmt. Die Regelungen waren unterschiedlich anspruchsvoll und erfassten immer nur Teilbereiche des Gewässerschutzes. Anforderungen an die Überwachung und Berichterstattung waren nicht harmonisiert. Als die Novellierung einiger alter Richtlinien und die Initiative zu einer neuen Gewässerökologierichtlinie anstanden, wurde die Kritik der Mitgliedstaaten und des Europäischen Parlaments unüberhörbar. Gefordert wurde ein schlüssiges Gewässerschutzkonzept, das die Kommission schließlich im Februar 1996 erstellte - die Grundlage der im Februar 1997 im Entwurf erstmals vorgelegten EG-Wasserrahmenrichtlinie (WRRL). Zahlreiche Änderungen erfolgten in der Diskussion mit den Mitgliedstaaten und dem Europäischen Parlament, bis die Richtlinie im Oktober 2000 verabschiedet wurde und am 22. Dezember 2000 in Kraft trat (1).

\section{- Wesentliche Neverungen}

Das Ziel eines umfassenden Gewässerschutzes geht die WRRL von mehreren Seiten an: Ihr Anwendungsbereich umfasst alle Gewässer der Gemeinschaft, einschließlich Grundwasser und Küstengewässer. Gleichzeitig führt sie eine Reihe von bisher nicht in dieser Klarheit oder Stringenz ein- gesetzten Konzepten der Wasserbewirtschaftung ein, die den nachhaltigen Umgang mit den Wasserressourcen fördern sollen:

- Die Einführung konkreter Umweltqualitätsziele,

- die Verankerung eines integrierten Flusseinzugsgebietsansatzes, der die grenzüberschreitende Dimension der Gewässerschutzpolitik betont, - die Einführung konkreter Planungsverfahren und -instrumente,

- die Betonung von Transparenz und Öffentlichkeitsbeteiligung sowie

- die Betrachtung der ökonomischen Dimension des Gewässerschutzes (2).

Außerdem werden alle gewässerrelevanten Einflussfaktoren berïcksichtigt, das heißt neben den Belastungen durch Nähr- und Schadstoffe auch Eingriffe durch Gewässerausbau und -aufstau bei Oberflächengewässern und mengenmäßige Eingriffe in den Grundwasserhaushalt. Wenn das Ziel des ,guten Zustands“ auf der Basis von Umweltqualitätsnormen und biologischen Parametern

\section{Der Umsetzungsplan der WRRL}

- Phase 1: Umsetzung in nationales Recht, Ausweisung der Flusseinzugsgebiete (bis Ende 2003),

- Phase 2: Charakterisierung der Flusseinzugsgebiete einschließlich der wirtschaftlichen Analyse (bis Ende 2004),

- Phase 3: Inbetriebnahme der Monitoringprogramme (bis Ende 2006),

- Phase 4: Veröffentlichung der Bewirtschaftungspläne einschließlich der Maßnahmenprogramme (bis Ende 2009),

- Phase 5: Umsetzung der Maßnahmenprogramme, Zielerreichung (bis Ende 2015). auch im Wesentlichen immissionsbezogen definiert ist, so sieht der so genannte kombinierte Ansatz der WRRL doch parallel dazu die Anwendung von Emissionsanforderungen und die Festlegung von Emissionswerten für prioritäre Stoffe vor. Der gute Zustand soll in allen Gewässern innerhalb von 15 Jahren erreicht werden (für den genauen Fahrplan siehe den Kasten). Darüber hinaus sind zwei Verlängerungen um je sechs Jahre möglich. Weitere Ausnahmemöglichkeiten lassen aber auch die Reduzierung der Ziele zu, wenn ökonomisch-technische oder natürliche Gegebenheiten der Zielerreichung entgegenstehen.

\section{Noch offene Fragen}

Auch wenn die WRRL teilweise sehr detaillierte Vorschriften enthält, sind eine Reihe von Fragen noch offen. Die in der Richtlinie bestehenden Interpretationsspielräume betreffen insbesondere methodisch-technische Fragen, deren Grundlagen zum Teil noch entwickelt werden oder deren Handhabbarkeit sich erst in der Praxis erweisen muss. Außerdem sollen drei Bereiche noch von Tochterrichtlinien geregelt werden: Zunächst war eine Liste von Stoffen zu erstellen, die ein erhebliches Risiko für die aquatische Umwelt darstellen. Dies ist im Dezember 2001 mit der Verabschiedung einer Liste 33 prioritärer Stoffe erfolgt (3), die als Annex X in die WRRL integriert werden. Nun sind bis Ende 2003 von der Kommission für diese Stoffe Vorschläge für Qualitätsnormen und Emissionsbegrenzungen vorzulegen. Für Grundwasser muss sie bis Ende 2002 Strategien zur Verhinderung und Begrenzung der Grundwasserverschmutzung vorlegen, die unter anderem Kriterien zur Beurteilung des guten chemischen Zustands und Verfahren für die flächenbezogene Bewertung enthalten. Schließlich hat sich die Kommission angesichts der zunehmenden und nicht harmonisierten Berichtspflichten selbst verpflichtet, die Berichtspflichtenrichtlinie bis 2004 zu revidieren.

\section{Gemeinsame Umsetzung in Europa}

In der Vergangenheit sind europäische Regelungen zum Umweltschutz zwar von den Mitgliedstaaten gemeinsam mit Kommission und Parlament erarbeitet worden, die Umsetzung wurde jedoch praktisch ausschließlich den Mitgliedstaaten überlassen. Die Folge waren teilweise unterschiedliche Auslegungen und Umsetzungsstrategien, die erst in Folge von Vertragsverletzungs- 
verfahren durch die Kommission oder Urteile des Europäischen Gerichtshofs gewissen Mindestanforderungen unterworfen wurden.

Zur Umsetzung der WRRL gehen die Mitgliedstaaten und die Europäische Kommission einen neuen, gemeinschaftlichen Weg - nicht zuletzt wegen der schlechten Erfahrungen mit der Umsetzung der bestehenden Gewässerschutzrichtlinien und der vielen offenen fachlichen und rechtlichen Fragen. Kurz nach der Verabschiedung der WRRL wurde von Vertretern der Mitgliedstaaten und Norwegens im Rahmen der informellen Treffen der „Wasserdirektoren“ die Notwendigkeit einer gemeinsamen Umsetzungsstrategie erkannt. Ein halbes Jahr später, im Mai 2001, konnte diese Strategie bereits beschlossen werden (4).

Ausgangspunkt für ein gemeinsames Vorgehen waren zunächst die übergreifenden Anforderungen, die die Bewirtschaftung von grenzüberschreitenden Flussgebieten mit sich bringt. Aber auch die Komplexität des Richtlinientextes, die Vielfalt möglicher Lösungen zu den angesprochenen wissenschaftlichen, technischen, rechtlichen und praktischen Fragen und der extrem kurze Zeitrahmen, der zur Umsetzung zur Verfügung steht, ließen eine gemeinsame Strategie sinnvoll erscheinen.

Die gemeinsame Umsetzungsstrategie bedeutet allerdings nicht, dass die Mitgliedstaaten aus ihrer Verpflichtung, die Inhalte der Richtlinie

\section{Abb. 1: Der gemeinsame europäische Umsetzungsprozess der Wasserrahmenrichtlinie (WRRL)}

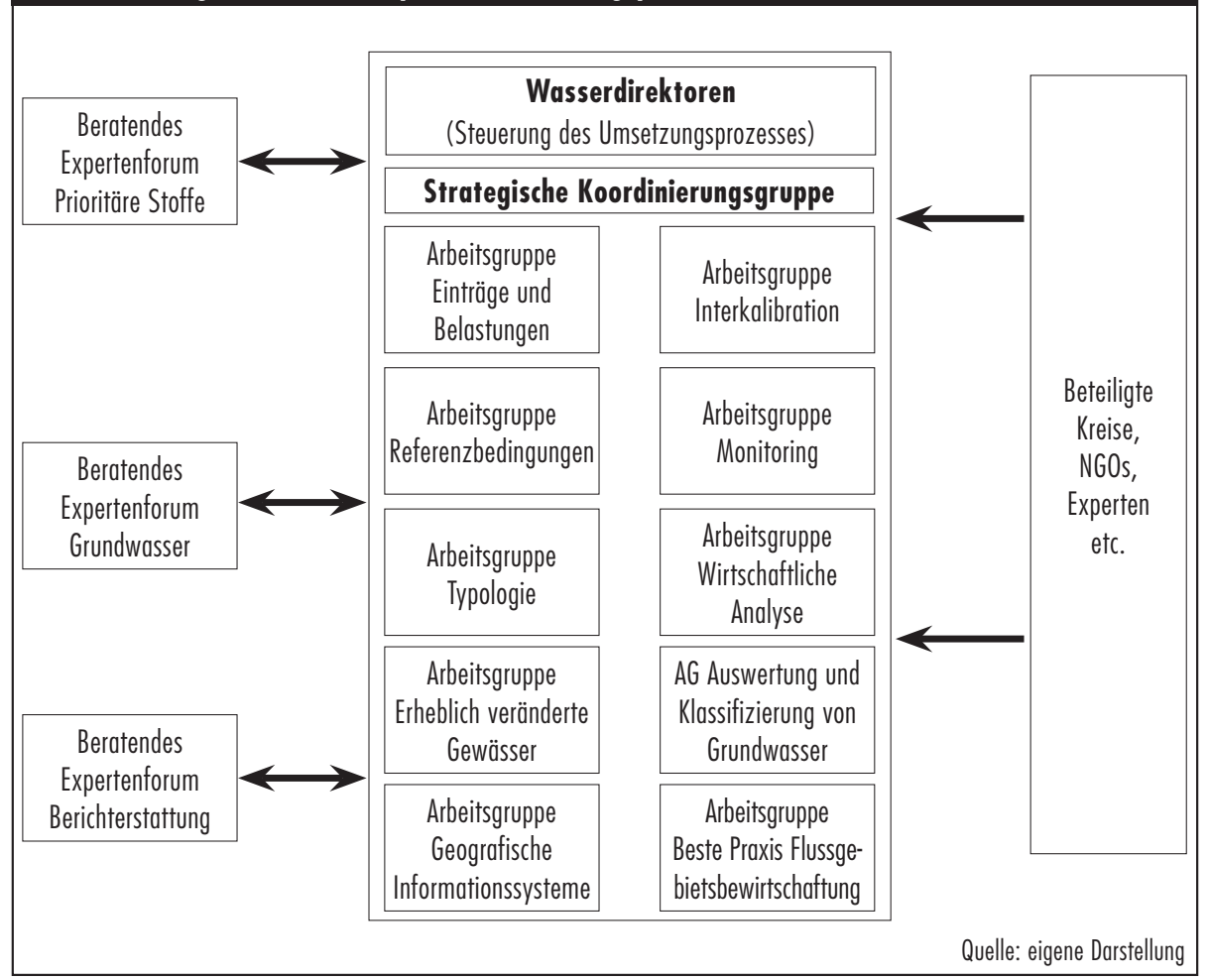

umzusetzen, teilweise entlassen sind. Vielmehr stellen die in diesem Rahmen getroffenen Absprachen nur Empfehlungen und Orientierungspunkte für die Mitgliedstaaten - und wohl auch für die Kommission bei der späteren Überprüfung - dar. Gerade an grenzüberschreitenden Flussgebieten sollen sie eine Grundlage für ein gemeinsames Handeln sein und helfen, Diskrepanzen zu vermeiden.

Kommission und Mitgliedstaaten haben thematische Arbeitsgruppen eingesetzt, die Leitlinien len. In Abbildung 1 ist dieser Prozess grafisch dargestellt. An allen Arbeitsgruppen sind nicht nur die Mitgliedstaaten und die Europäische Kommission, sondern auch die Beitrittskandidaten, Interessensvertreter der Wirtschaft sowie Umwelt- und Verbraucherverbände (NGOs) beteiligt. Letztere sind insbesondere in den $\mathrm{Ar}$ beitsgruppen zu „Einwirkungen und Belastungen“, zur ,wirtschaftlichen Analyse“ und zu den „erheblich veränderten Gewässern“, für die deutlich geringere Anforderungen gelten, präsent.

Parallel zu diesen Arbeitsgruppen arbeiten noch Expertengremien, die die oben genannten Tochterrichtlinien vorbereiten. Das entspricht eher dem üblichen Vorgehen bei der Erarbeitung europäischer Regelungen. Bemerkenswert sind jedoch inhaltliche Querbezïge. Probleme bei der Umsetzur Umsetzung bis Ende 2002 erarbeiten sol-

zung der Richtlinie, die von den Arbeitsgruppen erkannt werden, können daher bei der Vorbereitung der Tochterrichtlinien berücksichtigt werden.

\section{Die Umsetzung in Deutschland}

Die Konzeption der WRRL, alle Gewässer zukünftig in über Landes- und Staatsgrenzen hinausreichenden Flusseinzugsgebieten zu bewirtschaften, stellt für die meisten Mitgliedstaaten der EU auch eine große staatsorganisatorische Aufgabe dar. Lediglich in Großbritannien und Frankreich gibt es hier schon gewisse Erfahrungen. Für föderal gegliederte Staaten wie Deutschland ist bei der Umsetzung der Richtlinie insbesondere die verfassungsrechtliche Kompetenzverteilung zwischen Bund und Ländern zu beachten. Nicht immer können hier das fachlich Gebotene und das verfassungsrechtlich Erlaubte unter einen Hut gebracht werden.

Allerdings verlangt das EG-Recht auch nicht zwingend eine bundesrechtliche Umsetzung, sondern lediglich, dass die Richtlinienbestimmungen in den Mitgliedstaaten fristgerecht, flächendeckend, rechtsverbindlich und außenwirksam umgesetzt werden. Je nach Inhalt der Richtlinienbestimmung ist auch eine gewisse Einheitlichkeit der Umsetzung zu gewährleisten. Da dies allen an der Wassergesetzgebung Beteiligten in Bund und Ländern von Anfang an bewusst war, wurden bereits frühzeitig Foren und Arbeitsgruppen eingerichtet, die eine enge Zusammenarbeit sowie eine möglichst effektive und fristgerechte Umsetzung der WRRL auf allen Ebenen sicherstellen sollen.

Es wird parallel auf drei rechtlichen Ebenen gearbeitet: Die grundsätzlichen Anforderungen der Wasserrahmenrichtlinie sollen durch die siebte Novelle des Wasserhaushaltsgesetzes (WHG) in Bundesrecht überführt werden. Daneben müssen alle Landeswassergesetze novelliert werden. Die vielen fachlichen, sehr detaillierten Vorgaben, insbesondere der Anhänge II und V der WRRL werden zur Zeit in Form einer Musterverordnung vorbereitet, die dann von allen Bundesländern jeweils als Landesverordnung verabschiedet werden soll.

\section{Die siebte Novelle des WHG}

Nach ausfiuhrlichen Beratungen und Abstimmungen zwischen Bund und Ländern wird der Gesetzentwurf der Bundesregierung (5) zurzeit in Bundestag und Bundesrat behandelt. Die Novelle soll noch im Frühjahr 2002 verabschiedet werden.

Nach Art. 75 Grundgesetz steht dem Bund das Recht zu, Rahmenvorschriften über den Wasserhaushalt für die Gesetzgebung der Länder zu er- 
lassen. In Einzelheiten gehende oder unmittelbar geltende Regelungen sind nur in Ausnahmefällen zulässig. Dadurch ist es dem Bund verwehrt, eine umfassende und alleinige Regelung zur Umsetzung der WRRL im WHG zu treffen.

Was bedeutet dies nun konkret für die Umsetzung der WRRL? Durch die siebte WHG-Novelle werden nur die wesentlichen Eckpunkte, das heißt das Grundkonzept der WRRL, bundesrechtlich eingeführt. In erster Linie werden Regelungsaufträge an die Länder erteilt. Dennoch erfährt auch das WHG durch die WRRL eine neue Ausrichtung. Diese ist insbesondere durch den integrierten Ansatz einer Flusseinzugsgebietsbewirtschaftung mit dem Schwerpunkt auf Gewässerökologie gekennzeichnet.

Wie wird dieser neue Ansatz im WHG implementiert? Die Bewirtschaftung nach Flusseinzugsgebieten wird verankert; die zehn Gebiete - Donau, Elbe, Rhein, Oder, Maas, Ems, Weser, Eider, Schlei/Trave und Warnow/Peene - werden benannt und mit einer Karte im Anhang des Gesetzes anschaulich gemacht.

Die Zielbestimmungen der WRRL und deren Ausnahmen werden - der Struktur des geltenden WHG folgend - separat für die Bereiche Oberflächenbinnengewässer, Grundwasser und Küstengewässer eingeführt. Die umfassenden Planungsund Handlungsinstrumente der WRRL, das heißt das Maßnahmenprogramm und der Bewirtschaftungsplan, werden neu in das WHG aufgenommen. Sie ersetzen die bisherigen Planungsinstrumente Abwasserbeseitigungsplan, Reinhalteordnung und wasserwirtschaftlicher Rahmenplan sowie die bisherige Form der Bewirtschaftungspläne. Das Verfahren zur Aufstellung der Bewirtschaftungspläne einschließlich der Mitwirkung der Öffentlichkeit wird rahmenrechtlich fixiert.

\section{- Novellierung der Landeswasserge- setze}

Angesichts der komplexen Regelungsmaterie und der vielen offenen Fachfragen ist die Umsetzungsfrist der WRRL von drei Jahren relativ knapp bemessen. Deshalb bereiten die Bundesländer parallel zu den Beratungen zur Novelle des WHG bereits die Novellierung ihrer Landeswassergesetze vor. Auch dies geschieht in enger Abstimmung untereinander und mit dem Bund. Um ein Höchstmaß an Einheitlichkeit bei der Umsetzung der WRRL zu erzielen, wird von Gremien der Länderarbeitsgemeinschaft Wasser (LAWA) ein Musterentwurf erarbeitet und fortgeschrieben, das heißt an die jeweiligen Änderungen bei der WHGNovelle angepasst. Die konkrete Ausgestaltung der dort erteilten Regelungsaufträge liegt aber bei den Ländern. In den Landeswassergesetzen sind daher Vorschriften bzw. Verordnungsermächtigungen vorzusehen für die

- Zuordnung einzelner Gewässer zu Flussgebietseinheiten,

- Konkretisierung der Ziele und Anforderungen, - Festlegung der Fristen zur Zielereichung inklusive Verlängerungsmöglichkeiten,

- Bestandsaufnahme, Darstellung und Bewertung des Gewässerzustands,

- Gewässerüberwachung,

- Überwachung der Maßnahmen,

- Information und Anhörung der Öffentlichkeit bei der Erstellung der Bewirtschaftungspläne und

- für die Benennung der zuständigen Behörden. Die Grundlagen der Koordinierung der Bewirtschaftung in den Flussgebietseinheiten bereitet zur Zeit ein ad-hoc Arbeitskreis der LAWA vor. Generell wird eine Koordinierung der Arbeiten durch Verwaltungsabkommen der Länder untereinander als ausreichend, aber auch notwendig angesehen.

\section{Implementierung von fachlichen De- tails}

Die WRRL enthält insbesondere in ihren Anhängen II und V eine Vielzahl fachlicher Details zur Gewässerbeschreibung und zu den Anforderungen an die Gewässer, also zur Festlegung, Einstufung, Überwachung und Darstellung des Gewässerzustands. Diese würden einen Gesetzestext überfrachten, daher sollen diese Vorgaben in Verordnungsform in deutsches Recht umgesetzt werden.

Sicherlich wäre schon der Übersichtlichkeit und Einheitlichkeit wegen wünschenswert gewesen, eine Bundesverordnung zu erlassen. Wie sich jedoch schon am Beispiel der Umsetzung der EGGewässerschutzrichtlinie 76/464/EWG gezeigt hatte, stehen diesem Vorgehen verfassungsrechtliche Bedenken entgegen. Die Anhänge II und V der WRRL sollen deshalb in 16 Länderverordnungen auf der Grundlage einer von Bund und Ländern gemeinsam ausgearbeiteten Musterverordnung umgesetzt werden. Deren Entwurf ist bereits weit fortgeschritten und enthält neben einem Paragrafenteil mehrere ausführliche Anhänge. Zur Vollzugstauglichkeit werden die Anforderungen der WRRL an einigen Stellen weiter konkretisiert.

\section{Sonstige Umsetzungsmaßnahmen}

Neben der rechtlichen Umsetzung der WRRL darf nicht übersehen werden, dass viele fachliche Vor- gaben so angelegt sind, dass sie der weiteren wissenschaftlichen Erforschung und Begleitung bedürfen. Es handelt sich somit bei dieser Richtlinie nicht um ein fertiges Werk, sondern - wie schon der Name besagt - um einen ausgestaltungsbedürftigen Rahmen. Von wesentlicher Bedeutung ist, die auf den verschiedenen Ebenen gewonnenen wissenschaftlichen Erkenntnisse zusammenzufassen und für Vollzug und Öffentlichkeit bekannt zu machen. Zu diesem Zweck hat die LAWA unter anderem eine Arbeitshilfe erstellt (6). Darin stellt sie die rechtlichen Grundlagen, Hinweise für die Erstellung eines Bewirtschaftungsplans und weitere themenbezogene Arbeitspapiere dar. Mit dem Internetangebot WasserBLIcK (7) wird ein Informationsaustausch zwischen Behörden, aber auch für interessierte Bürger und NGOs eröffnet.

Damit sind die Voraussetzungen geschaffen, die mit der Umsetzung in Deutschland aufgrund der föderalen Struktur verbundenen rechtlichen und fachlichen Herausforderungen zu bewältigen.

\section{Anmerkungen}

(1) Richtlinie 2000/60/EG des Europäischen Parlaments und des Rates vom 23.10.2000 zur Schaffung eines Ordnungsrahmens der Gemeinschaft im Bereich der Wasserpolitik. Amtsblatt der Europäischen Gemeinschaften L 327/1 vom 22.12.2000

(2) Vgl. zu den neuen Impulsen auch Messner, F.: Chance und Herausforderung. Die neue Wasserrahmenrichtlinie der Europäischen Union. In: Ökologisches Wirtschaften, $\mathrm{Nr}$.

5/2000, S. 6-7.

(3) Entscheidung Nr. 2455/2001/EG des Europäischen Parlaments und des Rates vom 20.11.2001 zur Festlegung der Liste prioritärer Stoffe im Bereich der Wasserpolitik und zur Änderung der Richtlinie 2000/60/EG, Amtsblatt der Europäischen Gemeinschaften L 331/1 vom 15.12.2001.

(4) Strategic document, Common Strategy on the Implementation of the Water Framework Directive, April 2001.

(5) Die Bundestags-Drucksache 14/7755 vom 7.12.2001 enthält den Gesetzentwurf der Bundesregierung, die Stellungnahme des Bundesrates und die Gegenäußerung der Bundesregierung. (6) Länderarbeitsgemeinschaft Wasser: Arbeitshilfe zur Umsetzung der EG-Wasserrahmenrichtlinie, Stand: 20.02.2001, http://www.lawa.de

(7) Bund-Länder-Informations- und Kommunikationsplatfform zur EG-WRRL, http://wasserblick.net

\section{Die Autorlnnen}

Dr. Holger Brackemann, Dr. Christiane Markard und Dr. Jörg Rechenberg arbeiten in der Abteilung Wasser des Umweltbundesamtes (UBA).

Kontakt: UBA, Postfach 330022, 14191 Berlin, E-mail: wasser@uba.de 
(c) 20I0 Authors; licensee IÖW and oekom verlag. This is an article distributed under the terms of the Creative Commons Attribution Non-Commercial No Derivates License (http://creativecommons.org/licenses/by-nc-nd/3.o/), which permits unrestricted use, distribution, and reproduction in any medium, provided the original work is properly cited. 